\title{
Graphene oxide membrane for molecular separation: challenges and opportunities
}

\author{
Gongping Liu and Wanqin Jin
}

Membrane is a key component in systems such as battery, package, controlled delivery, solar cells and large-scale energy-efficient separation and purification processes [1]. In the last category, an engineered membrane controls the sorting of components by selective passage between feed and permeate streams. Such membrane separation technology plays an important role in reducing costs and environmental impacts of production of commodities at an order of magnitude less energy requirements compared with traditional approaches. Highly permeable and selective membranes are required to enable wider application of membrane technology. Polymeric membranes have dominated the practical application of membrane technology, while they generally suffer a trade-off between permeability and selectivity [2]. Since the 2010 Nobel Prize for "ground breaking experiments regarding the two-dimensional (2D) material graphene" [3], graphene-based materials have been used for a new family of membranes with extraordinary separation properties [4]. The atomic-thick graphene is able to minimize transport resistance and thus maximize permeate flux of the resulting membrane. Extraordinary molecular separation properties for purifying water and gases have been demonstrated by graphene-based membranes, which attract a huge surge of interest during last few years [5]. Graphene oxide (GO), the derivative of graphene, is most widely studied for membrane separation, since its oxygen-containing groups endow it with an excellent processibility and it can be assembled into ordered structures with nano- or even subnano-sized channels. This perspective highlights the latest creative studies in GO membranes and analyzes the barriers that must be overcome to further advance GO membranes for molecular separation.

The transport of molecules through laminar GO membrane usually occurs in in-plane slit-like pores and then plane-to-plane intergalleries [6]. Sometimes defects or pores of GO nanosheets provide diffusion shortcuts for molecules, or even exhibit molecular sieving properties by elaborately stacking these nanosheets [7]. The oxygencontaining groups on GO are able to form molecular interactions with water or gases such as $\mathrm{CO}_{2}$, which also contribute to the molecular transport through GO-based membranes [8]. A great number of studies demonstrate that the interlayer galleries between GO nanosheets play a vital role in fast and selective transport of water, ions and gases. How to precisely control the interlayer spacing is one of the key factors to achieve high-performance GO membranes.

It is challenging to reduce the interlayer spacing sufficiently to exclude small ions and maintain this spacing against swelling of GO membranes when immersed in aqueous solution, thereby limiting the potential in ion filtration applications. For instance, the use of GO membranes in water desalination is limited by a permeation cutoff of $\sim 9 \AA$ [9], which is larger than the diameters of hydrated ions of common salts. Nair and coworkers [10] proposed a physical confinement approach to control the interlayer spacing and thus achieve accurate and tunable ion sieving through GO membranes. They stored GO laminates strips under controlled humidity to produce a certain interlayer spacing and then embedded these GO laminates in epoxy to prevent further dimensional change when exposed to water. As a result, the interlayer spacing was reduced from 9.8 to $6.4 \AA$ with relative humidity changing from $100 \%$ to 0 , and the epoxy mechanically restricted the swelling of the GO laminates on exposure to humidity or liquid water. Ions permeation measurements $\left(\mathrm{K}^{+}, \mathrm{Na}^{+}, \mathrm{Li}^{+}, \mathrm{Ca}^{2+}\right.$ or $\mathrm{Mg}^{2+}$ ) showed an exponential decrease in ion permeation rate with decreasing interlayer spacing, but only a linear decrease in water permeation rate. This result is ascribed

State Key Laboratory of Materials-oriented Chemical Engineering, College of Chemical Engineering, Nanjing Tech University, Nanjing 210009, China * Corresponding author (email: wqjin@njtech.edu.cn) 

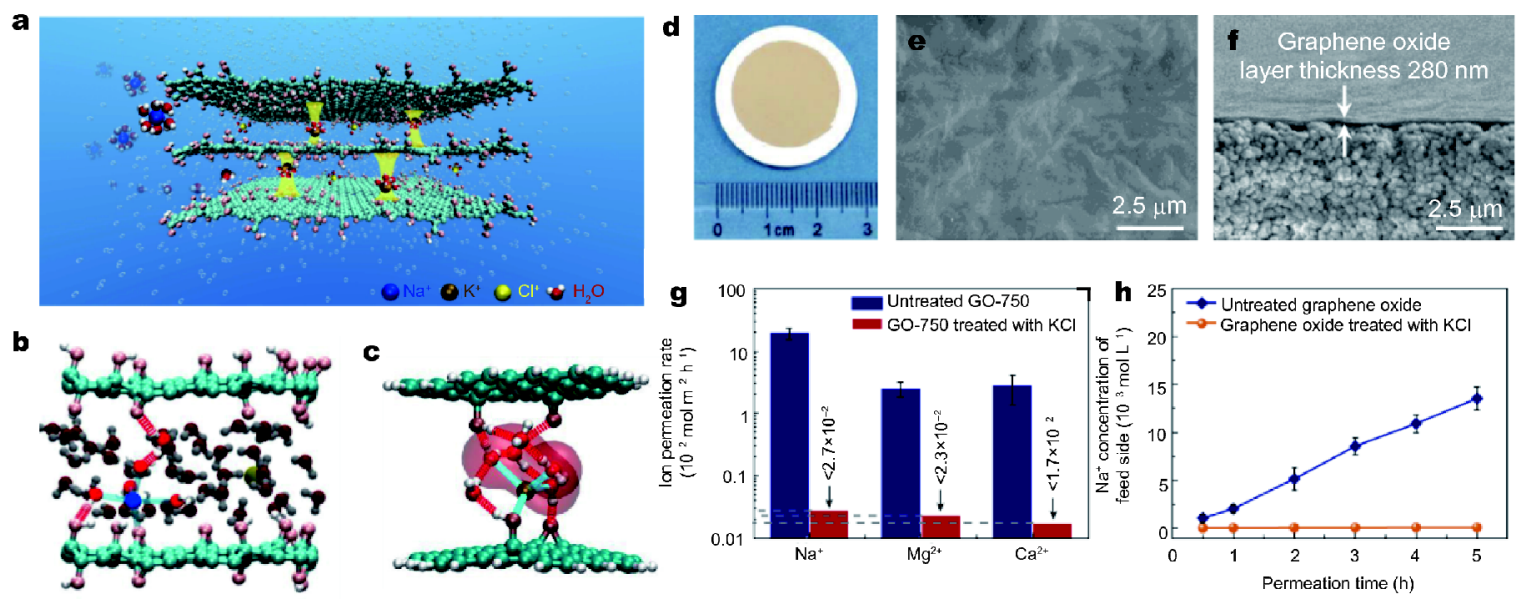

Figure 1 (a) A schematic of how $\mathrm{K}^{+}$ions in a GO membrane determines and fixes the interlayer spacing such that other cations are rejected while pure water can penetrate. Yellow pillars between the graphene oxide sheets depict the fixation of interlayer spacing by hydrated $\mathrm{K}^{+}$. (b) A snapshot of the $a b$ initio molecular dynamics simulation at 27 ps. Spheres in cyan, white, pink and red represent carbon, hydrogen, oxygen atoms in the oxygen functional group, and oxygen atoms in water molecules, respectively. Blue and yellow spheres represent $\mathrm{Na}^{+}$and $\mathrm{Cl}^{-}$; (c) the most stable optimized geometries of $\mathrm{K}^{+}-\left(\mathrm{H}_{2} \mathrm{O}\right)_{6} @ \mathrm{GO}$ clusters from density functional theory computation. The transparent red area is the van der Waals volume of the hydration water molecules, indicating a clear distortion of the hydrated structure of $\mathrm{K}^{+}$inside the GO sheets. (d) Digital photo; (e) surface SEM image and (f) cross-sectional SEM image of an $\mathrm{Al}_{2} \mathrm{O}_{3}$-supported GO membrane; (g) $\mathrm{Na}^{+}, \mathrm{Ca}^{2+}$ and $\mathrm{Mg}^{2+}$ permeation rates of untreated and $\mathrm{KCl}$-treated GO membranes, where dashed lines indicate the detection limits of the different cations; (h) $\mathrm{Na}^{+}$permeation rates of untreated and KCl-treated GO membrane with a thickness of about $280 \mathrm{~nm}$. Reproduced with permission from Ref. [11], Copyright 2018, Nature Publishing Group.

to the additional energy barriers that arise because of the necessity to partially strip ions from their hydrated shells so that they can fit inside graphene capillaries, while a low barrier for the entry of water molecules and large slip lengths inside the capillaries. However, scalable production of such membranes is difficult. Alternatively, they introduced this physical confinement idea into the synthesis of a composite membrane containing graphene flakes dispersed in GO laminates, in which the hydrophobic graphene resisted the GO swelling. The grapheneGO composite membrane shows a reduced interlayer spacing of $\sim 10.2 \AA$ compared with standard GO membranes of $\sim 14 \AA$ when immersed in water, thereby leading a $\mathrm{NaCl}$ rejection of $\sim 97 \%$ with a water flux of $\sim 0.5 \mathrm{~L} \mathrm{~m}^{-2} \mathrm{~h}^{-1}$ in forward osmosis measurement.

Recently, we reported a simple and scalable method to precisely control the interlayer spacing by using the ions themselves [11]. Cationic control of the interlayer spacing of $\mathrm{GO}$ membranes with ångström precision using $\mathrm{K}^{+}$, $\mathrm{Na}^{+}, \mathrm{Ca}^{2+}, \mathrm{Li}^{+}$or $\mathrm{Mg}^{2+}$ ions was demonstrated. Fixed interlayer spacing of GO membrane was obtained when immersed in ionic solutions $\left(\mathrm{KCl}, \mathrm{NaCl}, \mathrm{CaCl}_{2}, \mathrm{LiCl}\right.$ or $\mathrm{MgCl}_{2}$ ). Among them, the $\mathrm{KCl}$ solution produced the narrowest interlayer spacing of $11.4 \pm 0.1 \AA$, smaller than that immersed in pure water $(12.8 \pm 0.2 \AA)$. Moreover, $\mathrm{K}^{+}$ can stably and effectively fix the interlayer spacing at about $11 \AA$ even with the presence of equimolar cation
$\left(\mathrm{Na}^{+}, \mathrm{Ca}^{2+}, \mathrm{Li}^{+}\right.$or $\left.\mathrm{Mg}^{2+}\right)$ in the solution, endowing the rejection of other cations in mixed solutions (including $\mathrm{K}^{+}$itself). First-principles calculations reveal that the fixing of the interlayer spacing is mainly due to the interaction between the hydrated cations and aromatic rings (cation- $\pi$ interactions) on the GO nanosheet, as well as the interaction between the hydrated cations and the oxidized groups on the GO nanosheet (Fig. 1a-c). The GO membrane with cation-controlled interlayer spacing is successfully applied for sieving ions from water. Ion permeation tests of GO membrane supported by ceramic substrate indicate that the $\mathrm{KCl}$-controlled GO membrane exhibits efficient ion rejection (the reduction in ion permeation rate) of more than $99 \%$ relative to the pristine GO membrane (Fig. 1d-h). Water could still pass through the $\mathrm{KCl}$-controlled GO membrane with flux of 0.1$0.36 \mathrm{~L} \mathrm{~m}^{-2} \mathrm{~h}^{-1}$. Theoretical calculations show that cations such as $\mathrm{Fe}^{2+}, \mathrm{Co}^{2+}, \mathrm{Cu}^{2+}, \mathrm{Cd}^{2+}, \mathrm{Cr}^{2+}$ and $\mathrm{Pb}^{2+}$ have a much stronger cation $-\pi$ interaction with the graphene. Thus, other ions could be used to produce a wider range of interlayer spacings of GO membranes that can be applied for gas purification, solvent dehydration, lithium-based batteries and supercapacitors.

Structural stability is another critical issue for the application of GO membranes. The oxygen-containing groups on GO lead to undesirable redispersion of GO laminates in water, resulting in poor stability of GO 

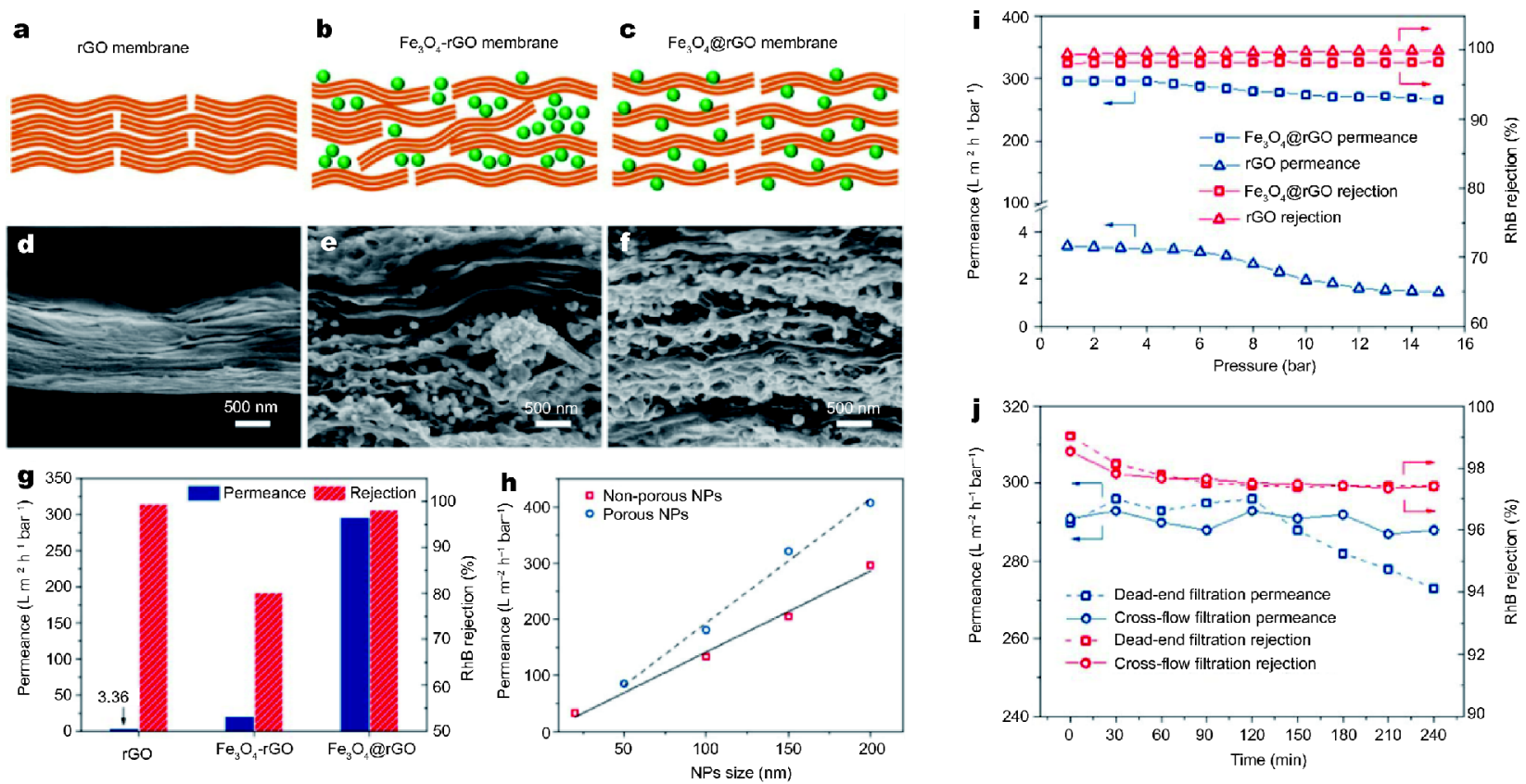

Figure 2 Schematic diagrams and SEM images of cross-sectional morphology of (a, d) rGO; (b, e) $\mathrm{Fe}_{3} \mathrm{O}_{4}-\mathrm{rGO}$; and (c, f) Fe $\mathrm{O}_{4} @ \mathrm{rGO}$ membranes; (g) water permeance and rejection of $\mathrm{rGO}, \mathrm{Fe}_{3} \mathrm{O}_{4}$-rGO, and $\mathrm{Fe}_{3} \mathrm{O}_{4} @ \mathrm{rGO}$ membranes; (h) water permeance of NPs@rGO membranes using nonporous $\mathrm{NPs}\left(\mathrm{Fe}_{3} \mathrm{O}_{4}, \mathrm{TiO}_{2}\right)$ or porous NPs (UiO-66) with different particle sizes; water permeance and RhB rejection of rGO and $\mathrm{Fe}_{3} \mathrm{O}_{4} @ \mathrm{rGO}$ membranes under (i) feed pressures from 1 to 15 bar and (j) dead-end/cross-flow operation (for $\mathrm{Fe}_{3} \mathrm{O}_{4} @ \mathrm{rGO}$ membrane only). Filtration conditions: feed: 50 ppm rhodamine B (RhB); pressure: 2 bar; temperature: $25^{\circ} \mathrm{C}$. Reproduced with permission from Ref. [12], Copyright 2017, Wiley-VCH.

membranes for water separation especially under crossflow in feed. Chemical crosslinking or reduction of GO could stabilize the membrane structure, while significantly decrease the water permeance. To this end, we proposed in-situ synthesis of nanoparticles (NPs) on GO nanosheets, in which chemical bonding was formed between NPs and GO, and in the meantime GO was reduced as rGO, thereby highly enhancing the structural stability of GO laminates in water [12]. Meanwhile, the uniformly grown NPs on GO nanosheets effectively enlarged the nanochannels to increase water permeance, meanwhile withstood the disordered laminar structure to preserve the selectivity, compared with pristine rGO membrane and conventional NPs intercalated GO membranes (Fig. 2). The resulting NPs@rGO membranes exhibit water permeance as high as $\sim 300 \mathrm{~L} \mathrm{~m}^{-2} \mathrm{~h}^{-1} \mathrm{bar}^{-1}$ and excellent rejections for organic dyes (e.g., $\mathrm{RhB}$ ) or heavy metal ions (e.g., $\mathrm{Cu}^{2+}$ or $\mathrm{Cd}^{2+}$ ) in water, as well as good stability under high pressure (up to 15 bar) and cross-flow filtration process. With this general NPs@rGO design, the water permeance can be easily tuned by varying the NPs size, and also can be further improved by in-situ growing nanoporous NPs to provide additional transport channels. The NPs@rGO membranes are filtrated on inner surface of commercial ceramic tubes, which provides a scalable strategy for the GO-based membranes as the tubes can be easily replaced by the state-of-the-art multi-channels products.

Considerable attention in water-based separation has been paid for GO membranes mainly due to ultrafast water permeation through low-friction graphene capillaries [13]. Fast and selective gas permeation through fewlayered GO membranes $[7,8]$ or GO incorporated polymer hybrid membranes [14] were also reported for separating gas pairs such as $\mathrm{H}_{2} / \mathrm{CO}_{2}$ and $\mathrm{CO}_{2} / \mathrm{N}_{2}$, in which subnanosized transport channels were provided by structural defects and/or interlayer galleries of GO nanosheets. We introduced external forces by using vacuum spinning technique combined with layer-by-layer molecular intercalation, to precisely manipulate the $2 \mathrm{D}$ channels of GO membranes for fast transporting and selective sieving gases [15]. The external forces are synergistic to regulate the stacking behavior of GO nanosheets so as to realize delicate size-tailoring of in-plane slit-like pores and plane-to-plane interlayer-galleries. The resulting GO membranes (EFDA-GO) with interlayer height of $\sim 0.4 \mathrm{~nm}$ exhibited 2-3 orders of magnitude higher $\mathrm{H}_{2}$ permeability and 3-fold enhancement in $\mathrm{H}_{2} /$ $\mathrm{CO}_{2}$ selectivity compared with commercial membranes (Fig. 3a). Recently, Yu and co-workers [16] fabricated 
a
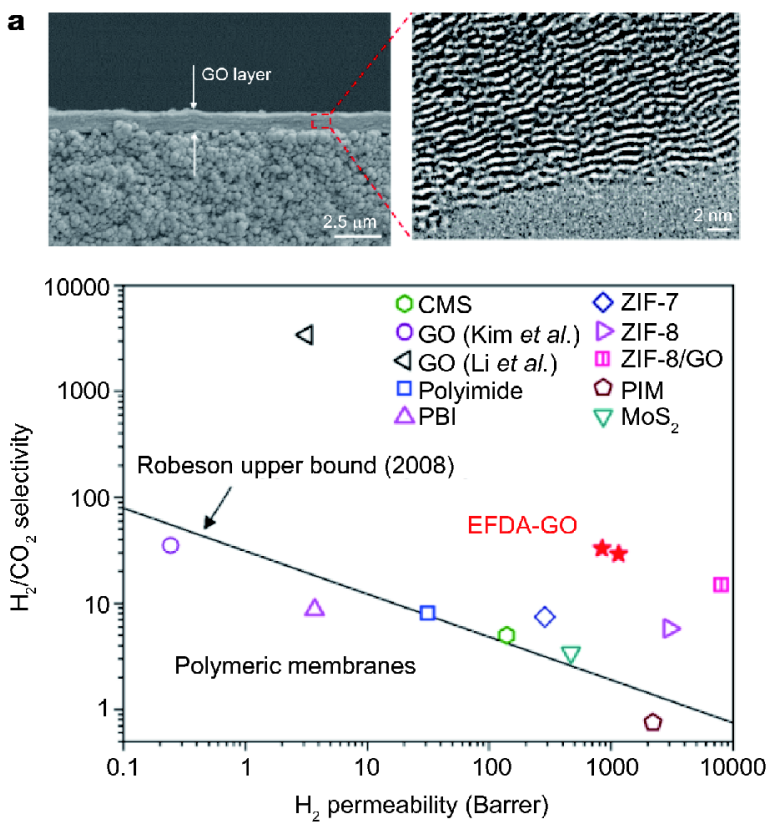

b
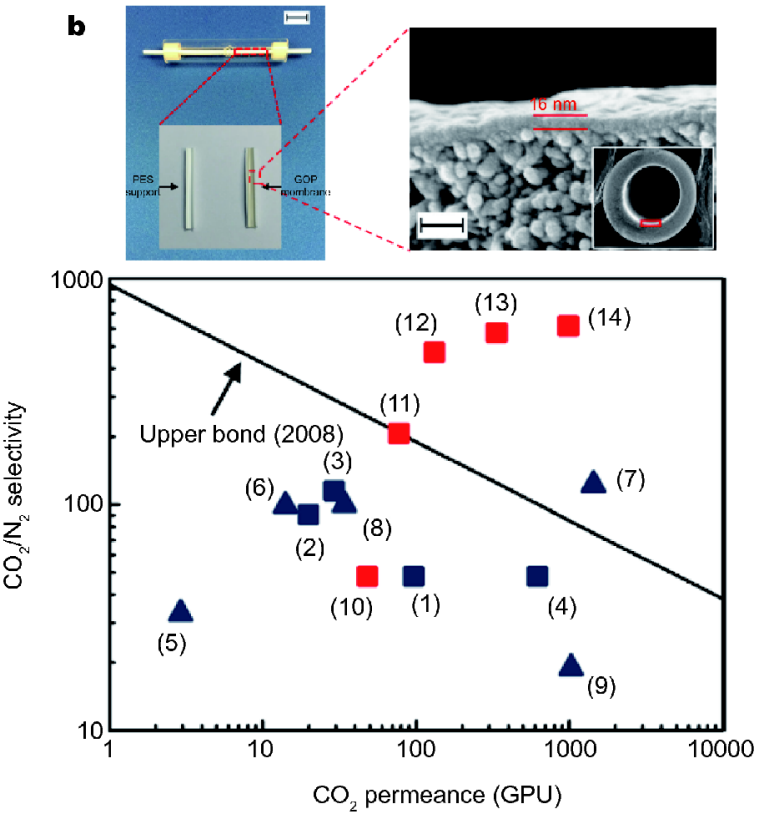

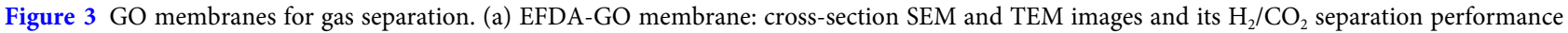
compared with literature. Reproduced with permission from Ref. [15], Copyright 2016, American Chemical Society. (b) GOP hollow fiber membrane: digital picture of a hollow fiber housed in a Pyrex tube. The scale bar is $10 \mathrm{~mm}$; inset shows the inner surface of PES support before (left) and after (right) depositing GOP coating. The effective separation length of the hollow fiber membrane is $43 \mathrm{~mm}$; the membrane was sealed in a Pyrex tube at two ends using epoxy, and two holes on each side were drilled into the Pyrex tube. The coated PES support surface shows a uniform dark gray color, suggesting a high-quality coating. Enlarged shows the cross-sectional SEM images of a GOP (10 wt.\% piperazine) membrane. The scale bar is 50 nm. Performance comparison of GOP membranes with polymeric membranes (black line) reported in 2008 upper-bound (modified upper bond (in GPU), thickness of polymer membranes is assumed to be $100 \mathrm{~nm}$ ) and some representative recent membranes. Squares denote the GO-based membranes; triangles denote the polymer-based membranes. (1) GO. (2) GO-Pebax. (3) GO-PEI. (4) GO-Borate. (5) Pebax-PEG-POSS. (6) PEI-Silica-Pebax. (7) PDMS. (8) PVAm. (9) PEG-based. (10) GO on single feed tests. (11) to (14) GOP at 24, 40, 60, 80 ${ }^{\circ} \mathrm{C}$, respectively, wet mixed feed. Reproduced with permission from Ref. [16], Copyright 2017, Nature Publishing Group.

ultra-thin $(<20 \mathrm{~nm})$ GO-based membranes on inner surface of polymeric (polyethersulfone) hollow fiber substrates by a vacuum filtration coating process. To promote $\mathrm{CO}_{2} / \mathrm{N}_{2}$ separation, a $\mathrm{CO}_{2}$-philic agent (piperazine) was introduced as a carrier-brush into the GO nanochannels with chemical bonding. The resulting amine-functionalized GO (GOP) hollow fiber membrane exhibited excellent $\mathrm{CO}_{2} / \mathrm{N}_{2}$ separation performance under simulated flue gas conditions with $\mathrm{CO}_{2}$ permeance of $1020 \mathrm{GPU}$ and $\mathrm{CO}_{2} / \mathrm{N}_{2}$ selectivity as high as 680 , showing great potential for practical $\mathrm{CO}_{2}$ capture process (Fig. $3 \mathrm{~b}$ ).

As an emerging area, organic solvent nanofiltration (OSN) using GO membranes [17] are received limited attention until recent work from Nair and co-workers [18] demonstrating precise molecular sieving and solvent permeation through ultra-thin, highly laminated GO membrane (HLGO). Large GO nanosheets with lateral size of $10-20 \mu \mathrm{m}$ were synthesized and used to fabricate the HLGO membrane, showing a narrower XRD peak compared to the conventional GO (CGO) membrane prepared from smaller nanosheets $(0.1-0.6 \mu \mathrm{m})$. The larger GO nanosheets contributed stronger interlayer interactions between larger overlapping areas, which is favored for eliminating occasional wrinkles and corrugation found in CGO membranes. As shown in Fig. 4, vacuum filtration of aqueous salt solution indicates that the HLGO, even as thin as $8 \mathrm{~nm}$, could block all ions with hydrated radii larger than $4.5 \AA$, whereas no molecular sieving is observed in the CGO membranes with a thickness of $8-50 \mathrm{~nm}$. It is interesting to see that the ultra-thin HLGO membranes are highly permeable to water and organic solvents, which seems contradictory to submicrometer-thick GO membranes that exhibit ultrafast water permeation but impermeable for solvents [13]. Moreover, the HLGO membranes exhibit $>99.9 \%$ rejection of small molecular weight organic dyes dissolved in methanol during OSN process. The observed organic solvent permeation and molecular sieving properties in the ultra-thin GO membranes are attributed to randomly distributed pinholes interconnected by short graphene 

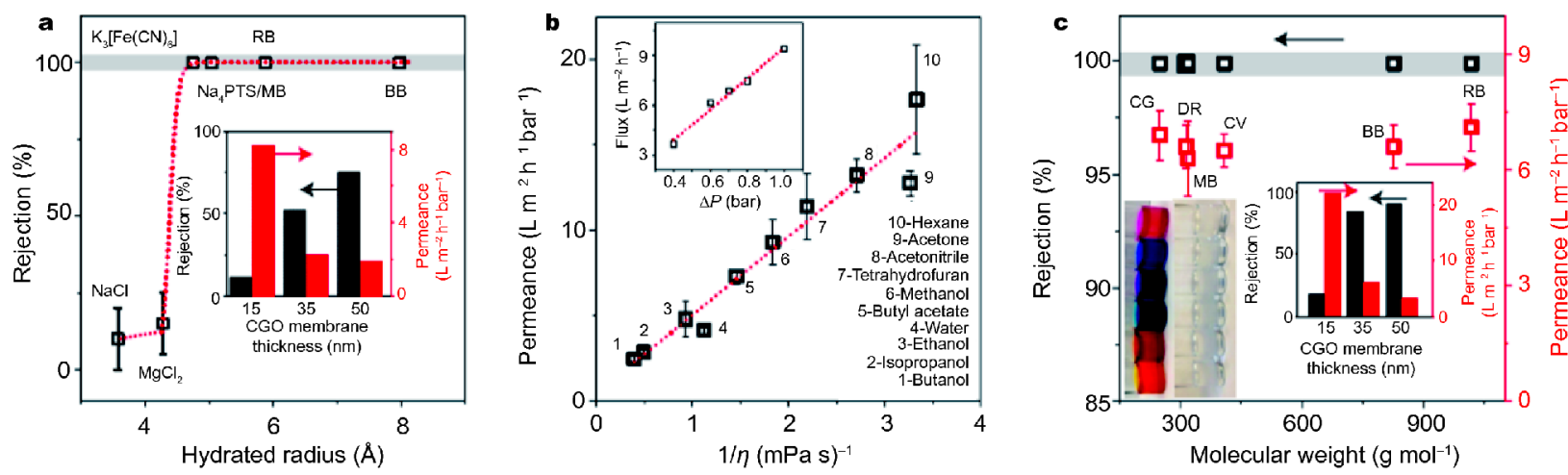

Figure 4 Molecular sieving and organic solvent nanofiltration through HLGO membranes. (a) Experiments for salt rejection as a function of the ion's hydrated radius. The HLGO membranes are $8 \mathrm{~nm}$ thick. MB, methylene blue; RB, rose Bengal; $\mathrm{BB}$, brilliants blue. Inset: MB rejection and water permeance exhibited by the CGO with different thicknesses. (b) Permeance of pure organic solvents through an $8 \mathrm{~nm}$ HLGO membrane as a function of their inverse viscosity. The used solvents are numbered and named on the right. Inset (top): methanol permeance as a function of pressure gradient. Dotted lines: best linear fits. (c) Rejection and permeance of several dyes in methanol versus their molecular weight. The dyes used: chrysoidine G (CG), disperse red (DR), MB, crystal violet (CV), BB and RB. Left inset: photographs of dyes dissolved in methanol before and after filtration through $8 \mathrm{~nm}$ HLGO membranes. Right inset: MB rejection and methanol permeance of CGO membrane with different thicknesses. Note that even though the dye rejection increases and approaches $\sim 90 \%$ with increasing CGO membrane thickness their permeance is significantly lower than $8 \mathrm{~nm}$ HLGO membranes. All the error bars are standard deviations using at least three different measurements from different samples. Points within the grey bar in a, $c$ show the rejection estimated from the detection limit. Reproduced with permission from Ref. [18], Copyright 2017, Nature Publishing Goup.

channels with a width of $1 \mathrm{~nm}$, rather than previously suggested interlayer capillaries in the sub-micrometerthick GO membranes [13].

In summary, the growing interest and achievements demonstrate the great potential and practical possibility of GO membranes for molecular separation. Key issues of developing GO membranes, precisely controlling the interlayer spacing and rationally improving the structural stability, have been well addressed by recent efforts. Fabrication of ultra-thin separation layer extends the application spectrum of GO membranes from water separation to gas separation and organic solvent nanofiltration. Efficient water desalination via pressure filtration process remains a challenge for GO membranes, demanding a robust membrane structure with permanent sub-nanosized channels. The transport pathways and mechanism through GO membranes still needs further investigations, in which atomic-level characterization techniques and theoretical models would be helpful. The insights gained in GO membranes might pave the avenue to a new family of high-performance separation membranes derived from other two-dimensional materials such as graphene-like MXene [19,20], $\mathrm{MoS}_{2}$ [21], nanoporous metal-organic framework (MOF) [22] and covalent organic framework (COF) [23] nanosheets.

Received 25 January 2018; accepted 10 April 2018; published online 28 April 2018

1 Koros WJ, Zhang C. Materials for next-generation molecularly selective synthetic membranes. Nat Mater, 2017, 16: 289-297

2 Park HB, Kamcev J, Robeson LM, et al. Maximizing the right stuff: The trade-off between membrane permeability and selectivity. Science, 2017, 356: eaab0530

3 Novoselov KS, Geim AK, Morozov SV, et al. Electric field effect in atomically thin carbon films. Science, 2004, 306: 666-669

4 Liu G, Jin W, Xu N. Two-dimensional-material membranes: a new family of high-performance separation membranes. Angew Chem Int Ed, 2016, 55: 13384-13397

5 Liu G, Jin W, Xu N. Graphene-based membranes. Chem Soc Rev, 2015, 44: 5016-5030

6 Han Y, Xu Z, Gao C. Ultrathin graphene nanofiltration membrane for water purification. Adv Funct Mater, 2013, 23: 3693-3700

7 Li H, Song Z, Zhang X, et al. Ultrathin, molecular-sieving graphene oxide membranes for selective hydrogen separation. Science, 2013, 342: 95-98

8 Kim HW, Yoon HW, Yoon SM, et al. Selective gas transport through few-layered graphene and graphene oxide membranes. Science, 2013, 342: 91-95

9 Joshi RK, Carbone P, Wang FC, et al. Precise and ultrafast molecular sieving through graphene oxide membranes. Science, 2014, 343: $752-754$

10 Abraham J, Vasu KS, Williams CD, et al. Tunable sieving of ions using graphene oxide membranes. Nat Nanotechnol, 2017, 12: 546-550

11 Chen L, Shi G, Shen J, et al. Ion sieving in graphene oxide membranes via cationic control of interlayer spacing. Nature, 2017, 550: $380-383$

12 Zhang M, Guan K, Shen J, et al. Nanoparticles@rGO membrane enabling highly enhanced water permeability and structural stability with preserved selectivity. AIChE J, 2017, 63: 5054-5063

13 Nair RR, Wu HA, Jayaram PN, et al. Unimpeded permeation of water through helium-leak-tight graphene-based membranes. Science, 2012, 335: 442-444

14 Shen J, Liu G, Huang K, et al. Membranes with fast and selective 
gas-transport channels of laminar graphene oxide for efficient $\mathrm{CO}_{2}$ capture. Angew Chem Int Ed, 2015, 54: 578-582

15 Shen J, Liu G, Huang K, et al. Subnanometer two-dimensional graphene oxide channels for ultrafast gas sieving. ACS Nano, 2016, 10: 3398-3409

16 Zhou F, Tien HN, Xu WL, et al. Ultrathin graphene oxide-based hollow fiber membranes with brush-like $\mathrm{CO}_{2}$-philic agent for highly efficient $\mathrm{CO}_{2}$ capture. Nat Commun, 2017, 8: 2107

17 Huang L, Chen J, Gao T, et al. Reduced graphene oxide membranes for ultrafast organic solvent nanofiltration. Adv Mater, 2016, 28: 8669-8674

18 Yang Q, Su Y, Chi C, et al. Ultrathin graphene-based membrane with precise molecular sieving and ultrafast solvent permeation. Nat Mater, 2017, 16: 1198-1202

19 Ding L, Wei Y, Li L, et al. MXene molecular sieving membranes for highly efficient gas separation. Nat Commun, 2018, 9: 155

20 Liu G, Shen J, Liu Q, et al. Ultrathin two-dimensional MXene membrane for pervaporation desalination. J Membrane Sci, 2018, 548: $548-558$

21 Wang Z, Tu Q, Zheng S, et al. Understanding the aqueous stability and filtration capability of $\mathrm{MoS}_{2}$ membranes. Nano Lett, 2017, 17: 7289-7298

22 Peng Y, Li Y, Ban Y, et al. Metal-organic framework nanosheets as building blocks for molecular sieving membranes. Science, 2014, 346: 1356-1359

23 Li G, Zhang K, Tsuru T. Two-dimensional covalent organic framework (COF) membranes fabricated via the assembly of exfoliated COF nanosheets. ACS Appl Mater Interfaces, 2017, 9: 8433-8436

Acknowledgements This work was financially supported by the National Natural Science Foundation of China (21490585, 21476107, 21776125 and 51861135203), the Innovative Research Team Program by the Ministry of Education of China (IRT17R54) and the Topnotch Academic Programs Project of Jiangsu Higher Education Institutions (TAPP).

Author contributions Liu G and Jin W wrote the paper, and contributed to the general discussion.

Conflict of interest The authors declare no conflict of interest.

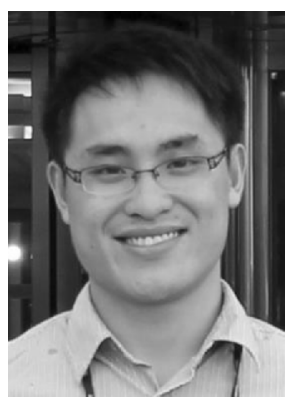

Gongping Liu is an associate professor in Nanjing Tech University. After receiving his $\mathrm{PhD}$ degree under the supervision of Professor Wanqin Jin at Nanjing University of Technology in 2013, he joined Nanjing Tech University as an assistant professor. During 2015-2017, he worked as a postdoctoral fellow in Professor William J. Koros group at Georgia Institute of Technology. His current research focuses on advanced membranes based on mixed-matrix formulation and 2D materials, with applications in molecular separations including gas separation, organic compounds recovery and purification.

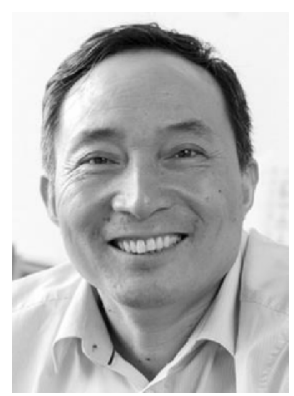

Wanqin Jin is a professor of chemical engineering at Nanjing Tech University. He received his PhD degree from Nanjing University of Technology in 1999. He was a research associate at the Institute of Materials Research \& Engineering of Singapore (2001), an Alexander von Humboldt Research Fellow (2001-2013), and visiting professors at Arizona State University (2007) and Hiroshima University (2011, JSPS invitation fellowship). His current research focuses on membrane materials and processes. He has more than 300 publications with over 9,500 citations and H-index of 56. He severed as an Editor of Journal of Membrane Science.

\section{用于分子分离的氧化石墨烯膜: 机遇与挑战}

刘公平, 金万勤

摘要 氧化石墨烯作为一种典型二维材料具有单原子层厚度和大量含氧官能团, 可作为高性能分离膜的理想构筑单元，已成为近年来的 研究热点. 通过对氧化石墨烯纳米片的有序组装, 可构筑亚纳米尺寸的分子传质通道, 对水分子、有机小分子、气体分子具有快速选择性 透过效应, 在水处理、溶剂纯化和气体分离等膜过程展现出优异的分离性能. 本文重点分析了近期氧化石墨烯膜的突破性进展, 并指出氧 化石墨烯膜用于分子分离过程所面临的机遇与挑战. 